\section{A Proposal for increasing the Beam Output from a Synchrocyclotron}

IN this communication, the possibility is considered of appreciably increasing the mean output of a synchrocyclotron, using relatively inexpensive equipment and with the expenditure of only a small amount of electrical power.

Suppose that a small dee system, covering the orbits of ions with energies of up to about $5 \mathrm{MeV}$., is placed opposite the live dee at the centre of the machine. To this small additional dee a high-voltage radio-frequency signal is fed which is in antiphase to that on the main dee. This signal is derived from the main oscillator system after amplification in a separate power amplifier. The auxiliary amplifier is pulsed on at a time slightly before the main dee commences to capture ions from the ion source, and is switched off when the captured ions have gained on the average a few MeV., that is, when the ion bunch is circulating at a radius such that it truly enters the main dee electrode and thus picks up the full dee voltage. It is essential that the auxiliary dee signal is reduced to a very small value as the ion bunch approaches the radius of the small dee edge, to avoid inducing radial betatron oscillations.

In Fig. 1 the assumed variation of the energy gained per turn by an ion is shown as a function of the orbit radius. Three cases are shown: the first curve corresponds to a normal synchrocyclotron, and the second curve to a machine modified in the way here described. The third curve, produced by too large a voltage on the small dee, is probably undesirable, because ions captured into stable orbits at the peak of the curve would afterwards be lost. Obviously, to achieve the required shape of the curve, the auxiliary power amplifier output must be gradually tapered off.

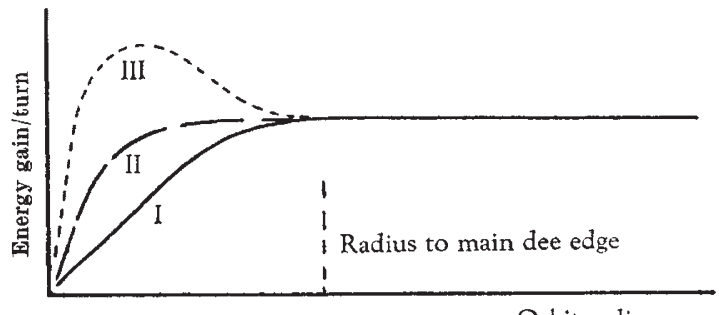

Fig. 1

The manner in which such an arrangement could produce an increase in the intensity of the beam is now considered. First, the energy gained per turn by the ions would be increased, permitting the use of a larger area in phase-space for ion capture. Secondly, the ions would be extracted more rapidly from the region of high particle density near the ion source, with a consequent reduction in the number of ions lost by gas scattering. Thirdly, the production of a more uniform electric field in the central region would cause a reduction in the movements of the centres of the orbits away from the centre of the machine, thereby reducing the amplitudes of the radial betatron oscillations.

The band-width and power requirements of the auxiliary amplifier are quite modest; a power of 1-2 per cent of the main oscillator power is sufficient, and the band-width need only cover the range of synchronous frequencies required by the ion bunch

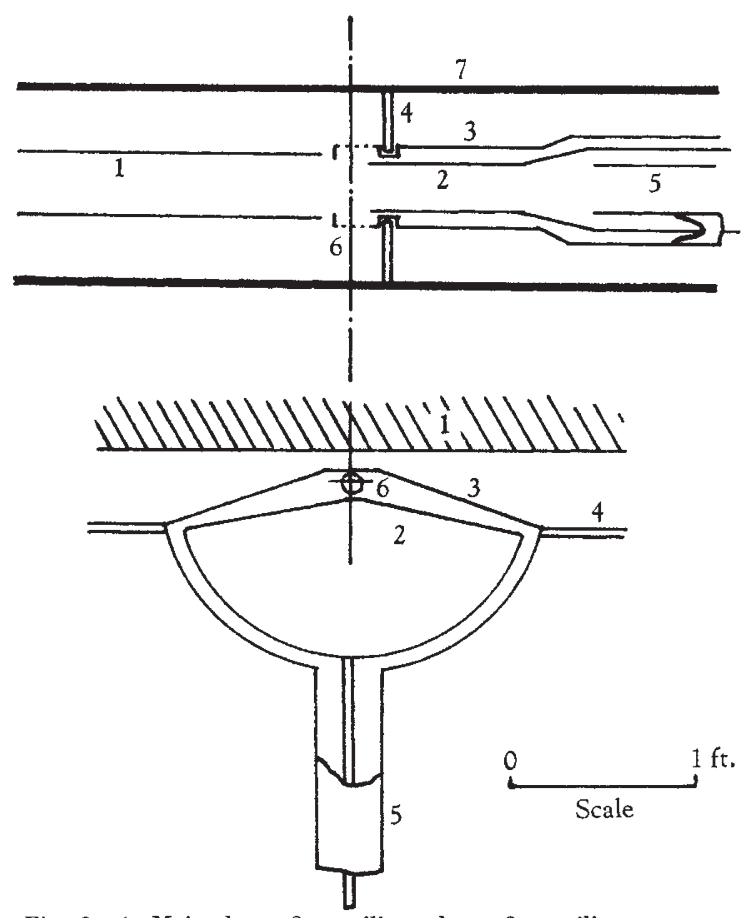

Fig. 2. 1, Main dee ; 2 , auxiliary dee ; 3 , auxiliary screen ; 4 , dummy dee ; 5 , co-axial feeders $; 6$, hole for ions ; 7 , vacuum

in its passage from the source to the radius at which the auxiliary amplifier is switched off. So that large signals are not induced in the small dee system by the main dee when it is switched off, the feed line should be resistively loaded; but this is usually also required to achieve a sufficiently wide band-width. In addition, a small earthed sereen between the main dee and the auxiliary dee is desirable to reduce the electric coupling between the two dees and to improve the shape of the field near the centre of the machine. A typical arrangement is shown in Fig. 2.

Preliminary tests of this scheme have been made with the Harwell 110-in. synchrocyclotron, using an 11-in. radius dee with a total operating time of approximately 50 usec. in each main modulation cycle. The rate of repetition for this machine is $\mathbf{2 0 0}$ c./s. A peak voltage of $4 \mathrm{kV}$. was used on the small dee, the main dee voltage being approximately $8 \mathrm{kV}$. peak. Despite a reduction in the vertical aperture of 25 per cent, caused by the small dees, an increase in the beam current measured at $50 \mathrm{meV}$. of approximately 60 per cent was readily achieved.

I thank the members of the team engaged on this work, in particular P. S. Rogers, for their help, and also J. D. Lawson for helpful discussions. F. M. Russell

Rutherford High Energy Laboratory, National Institute for Research in Nuclear Science, Harwell, Didcot, Berks.

\section{Effect of a Resisting Couple on the Rotational Motion of a Rigid Body}

IN a recent communication ${ }^{1}$, Nonweiler demonstrates the proposition that an external couple applied to a rigid body about its instantaneous axis of rotation in the sense opposing its rotation tends to cause the axis to approach the axis of greatest 